\title{
Living the DREAM: A Milestone in Canadian Research
}

\section{Jonathon Torchia}

(University of Toronto)

News Reporter (HSI 2011-2012)

Recently, two researchers at McMaster University completed the first human clinical trial to investigate preventative drugs in the fight against diabetes.

There is no doubt that diabetes is one of the most pressing health concerns of our generation, as more than 9 million Canadians live with diabetes or pre-diabetes (Canadian Diabetes Association). Treatment of diabetes through therapeutic intervention is a multi-billion dollar industry, and is estimated to cost the Canadian health care system $\$ 16.9$ billion per year by 2020 .

\section{In the past decade and over the course of around 9 trials we have clearly shown that diabetes can be prevented.}

Canada is a leader in advancing basic molecular science and clinical research in diabetes. Recently, researchers have become interested in disease prevention, as opposed to simply recurrent drug therapy, in order to lessen the burden on Canada's overwhelmed health care system.

In 2006, the DREAM trial (Diabetes REduction Assessment with ramipril and rosiglitazone Medication), co-led by Dr. Hertzel Gerstein and Dr. Salim Yusuf at McMaster University, was completed as the first clinical trial to investigate diabetes prevention as the primary end-point (available online through PubMed; ID16997664). The study showed that type 2 diabetes can be prevented by up to $60 \%$ with the use of rosiglitazone, and has put the thiazolidinedione (TZD) drugs on the radar as promising drugs for the prevention of type 2 diabetes. The following is a brief interview with $\mathrm{Dr}$. Hertzel Gerstein, highlighting his personal opinions about the state and influence of Canadian diabetes research.

JT: There is an opinion amongst many people that type 2 diabetes is a "man-made" disease, and that the burden placed on Canada's health care system can be entirely prevented by maintaining a healthy lifestyle. Is this true?

It's not a self-induced disease. People don't cause their own diabetes and you are not responsible for the diseases that you get; in the same way that you are not responsible for your lung cancer if we happen to live in a polluted environment. You are not responsible for radiation poisoning if you happen to live next to a leaky nuclear facility. People do not cause their diabetes, they do not cause their diseases, and it would be completely wrong to suggest so. The environment that we live in is a very 'diabetogenic' environment and induces the disease of the people who live within it. Our responsibility is to respond to the challenges of the day.

JT: How would you describe the support that Canada has put forth in terms of tackling the challenges of our day? Is there adequate support available to conduct the necessary research?

Diseases are international and the problem is international. Canadian researchers have a long, distinguished history in diabetes research in the past 100 years. The discovery of insulin [at the University of Toronto] is the most famous of these but also the first implementation of islet cell transplantations and the discovery of multiple other hormones/drugs were pioneered by Canadians. The history of success in Canada is large, ranging from the genetic level, therapeutic level and diagnosis advancements. Canada is blessed to have such a large number of researchers on this topic and that is fantastic. The funding environment for Canadian researchers is not optimal, but in fact, Canada consistently punches in well above its weight in terms of the world stage internationally, and the productivity is well above what is funded.

JT: Describe the coming together of the "DREAM team" and its main goals and visions.

At the time there were no clear ways to prevent diabetes. There were suggestions from the HOPE trial that other 


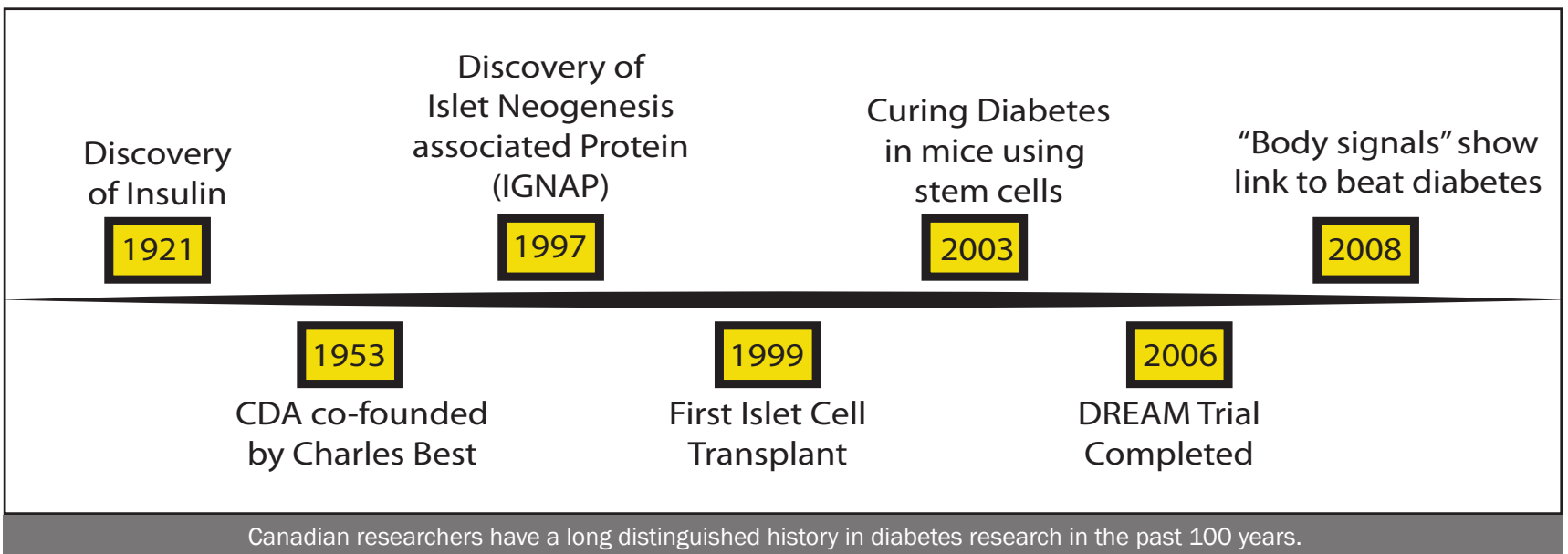

drugs like the ACE inhibitors might prevent diabetes. The HOPE trial data suggested Ramipril might be preventing the outcome of diabetes. There was also interest in TZD drugs. The opportunity [for collaboration with renowned leaders in the field] arose and the proposal led to a consortium of funding from the Canadian Institutes of Health Research (CIHR) as well as by three different pharmaceutical companies in a beautiful example of industry-government collaboration, and the rest is history...

\section{JT: What follow-up experiments have spawned from the DREAM trial results?}

We are still interested in diabetes prevention and whether novel therapies reduce the risk of diabetes. The DREAM trial clearly showed that TZD drugs could reduce the risk of diabetes by $60 \%$ and we have led a number of studies investigating other treatment options such as the combination of TZD drugs with Metformin. In the past decade and over the course of around 9 trials, we have clearly shown that diabetes can be prevented. We weren't solely interested in the drug itself [rosiglitazone] but the class, the TZD class of drugs and their effect on [PPAR] gamma-activation that was promising. One [follow-up] study in particular is a large trial of 12,500 people with pre-diabetes or early diabetes and how the use of insulin can actually prevent cardiovascular problems, of which the results will be available in the next 8 months.

Dr. Gerstein then informs us that there is never a failed trial. Each study brings with it a set of new questions and new hypotheses that build upon previous results, a notion that forms the foundation of the scientific method. While the use of rosiglitazone has been met with recent controversy concerning potential cardiovascular toxicity, the TZD class as a whole remains of primary interest. And as mentioned, further studies that address these issues are underway and near completion. The DREAM trial showed us that type 2 diabetes can be prevented with an efficacy comparable to the gold standard of a complete life-style change, a feat not many drugs can claim to their name. It will certainly be interesting to compare results of other TZD chemical analogs to the efficacy of the commonly used rosiglitazone.

As Dr. Gerstein has reminded us, we Canadians are in good hands. It is clear that the maple leaf nation is at the forefront of scientific inquiry. Dr. Gerstein reminds us that "You can't look backwards to create the future and the only way to do that is to foster an environment that supports research. That is, empirical inquiry and investigation into what nature can and can't do. If our society ever abandons that, we are doomed, and we will drift away and die."

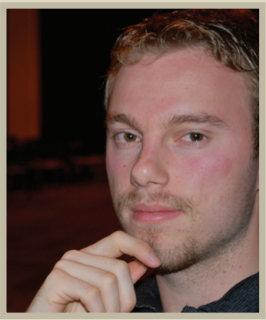

\section{Jonathon Torchia}

Jonathon Torchia completed both undergraduate and Master's biochemistry degrees at McMaster University before pursuing a PhD at the University of Toronto in the Department of Laboratory Medicine and Pathobiology (LMP). In his Master's work, he studied anti-cancer natural product biosynthesis from various bacteria, using next generation genome sequencing. In Jonathon's doctoral work, he will be extending his bioinformatics skills to study the genetics and molecular mechanisms of rare peadiatric brain tumors. 